\title{
Periodic fluctuations in synaptic transmission and enhancement of transmission in hippocampal slices
}

\author{
WALTER C. LOW*, SPENCER L. BEMENT and DAVID WHITEHORN \\ Bioelectrical Sciences Laboratory, University of Michigan, Ann Arbor, MI 48109 and Department of \\ Physiology and Biophysics, University of Vermont, Burlington, VT 05405 (U.S.A.)
}

(Accepted October 8th, 1981)

Key words: periodic fluctuations - synaptic transmission - hippocampal slices

The properties of the synchronously activated radiatum fiber-CAl synaptic population were examined with the in vitro hippocampal slice preparation. Periodic fluctuations in synaptic transmission and in the enhancement of synaptic transmission were observed with periods ranging from 8 to $20 \mathrm{~s}$. Such periodic fluctuations did not arise from fluctuations in afferent radiatum fiber activity. The period and amplitude of the cyclic variations in the enhancement of synaptic transmission were found to be altered with repeated electrical stimulation of the radiatum fibers. These results reflect cooperative synaptic actions which must be taken into consideration in the delineation of the mechanisms of potentiation.

The statistical nature of neurotransmission is now well documented ${ }^{3,6,13}$. Recent studies, however, using frog neuromuscular junction and electric organ of Torpedo marmorata $^{4,5}$ suggest that slow oscillations in the availability and release of neurotransmitters may also occur. We now present evidence for periodic fluctuations in synaptic transmission and in the enhancement of synaptic transmission for a homogeneous population of synapses within the mammalian central nervous system. In hippocampal slices from rat we recorded evoked field potentials that reflect afferent fiber responses and summated dendritic depolarizations (population EPSPs) evoked by the electrical stimulation of afferent radiatum fibers that terminate on the apical dendrites of CAI pyramidal cells $\mathrm{s}^{2,8,12}$. With stimuli delivered at a rate of $0.5 \mathrm{~Hz}$ we found a well developed periodicity in the size of the population EPSP, but not in the afferent radiatum fiber response. With paired stimuli (40 ms interstimulus interval) the response to the second stimuli was generally greater than the first. This facilitation was quantified as a test:control ratio. We found a strong periodicity in the magnitude of the test:control ratios with the period of the oscillations ranging from 8 to $20 \mathrm{~s}$ in different preparations. In any particular slice the periodicity for test:control ratios was similar to that observed for responses to single stimuli. Thus a regular oscillation appears to exist both in synaptic transmission and in the facilitation of transmission which occurs as a result of prior activation.

Hippocampal slices were prepared from rats (Sprague-Dawley, 100-125 g) with methods similar to those previously described ${ }^{7,8}$. Slices were sectioned transverse to

\footnotetext{
* Present address: Department of Physiology and Biophysics, University of Vermont, Burlington, YT 05405, U.S.A.
} 


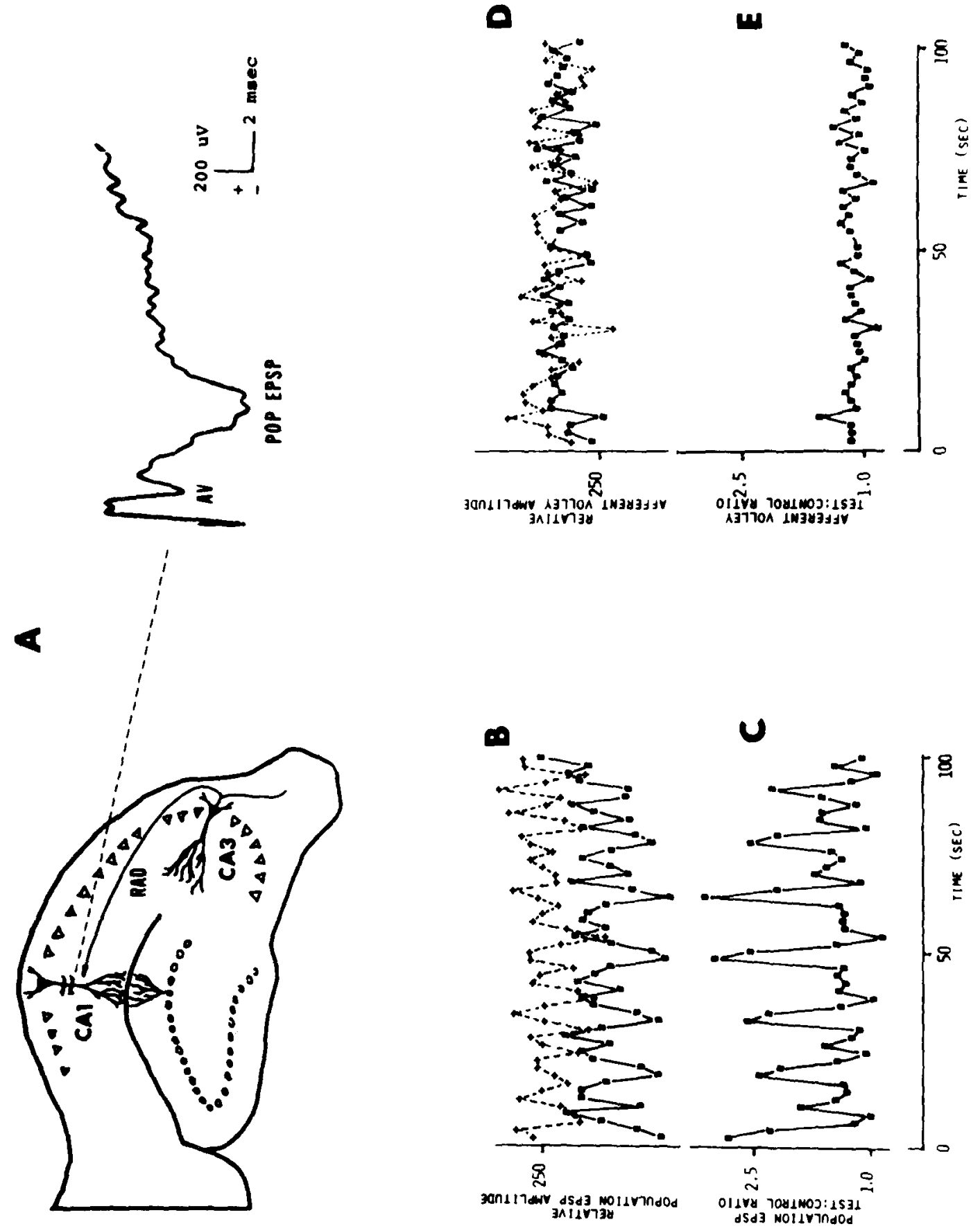

Fig. 1. Oscillation of cvoked population EPSP responses. A: afferent volley (AV) and population EPSP as recorded in stratum radiatum in response to a single stimulus applied to radiatum fibers. $B$ : control (squares) and test (crosses) population EPSP amplitudes in response to paired stimuli ( $40 \mathrm{~ms}$ inter-stimulus interval) applied once every $2 \mathrm{~s}$ with observed oscillations of both test and control population EPSPs. The period of oscillation for the test responses is $8 \mathrm{~s}$. The oscillation for the control responses appear to exhibit two frequency components, one with a period of $8 \mathrm{~s}$ and the other with a period of $16 \mathrm{~s}$. Note the phase relationship $\left(\sim 180^{\circ}\right)$ between the control and test responses. $\mathrm{C}$ : oscillation of the test:control ratio with a major period of approximately $16 \mathrm{~s}$. The large excursion in this ratio reflects the phase relationship noted in Fig. 1B. D: oscillation of test (crosses) and control (squares) afferent volleys. Periodicities are not as distinct as those of the population EPSP and fluctuations appear more random in nature. E: fluctuation of afferent volley test:control ratio. Note that these fluctuations do not exhibit oscillations like that found for the population EPSP in Fig. $1 \mathrm{C}$. 
the septo-temporal axis of the hippocampal formation and immediately immersed in a cooled $\left(\sim 10^{\circ} \mathrm{C}\right)$ and aerated glucose Ringer solution $(124 \mathrm{mM} \mathrm{NaCl}, 5 \mathrm{~m} \mathrm{M} \mathrm{KCl}, 1.24$

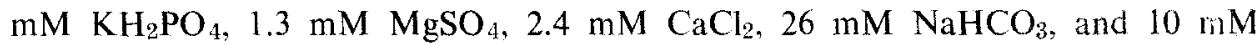
glucose). After sectioning, slices were placed upon a nylon net in the inner well of a warmed $\left(37+0.5^{\circ} \mathrm{C}\right)$ incubation chamber that also contained the glucose Ringer. $A$ gaseous mixture of $95 \% \mathrm{O}_{2}$ and $5 \%, \mathrm{CO}_{2}$ was bubbled into the outer well of the chamber filled with distilled water for approximately $2 \mathrm{~h}$ to create an oxygen gradient which results in the diffusion of $\mathrm{O}_{2}$ into the slices.

Bipolar stimulating electrodes (40-gauge Nichrome) were placed in stratum radiatum of subfield $\mathrm{CAl}$, and an isolated constant current source driven by a Grass stimulator (Model SD5) was used to excite the radiatum fibers with paired current pulses separated in time by $40 \mathrm{~ms}$. Current pulses of $100 \mu$ s duration were used to evoke responses from strata radiatum. The range of current intensities used for the majority of the slices typically varied from 30 to $100 \mu \mathrm{A}$. A recording electrode (1- 3 $\mathrm{M} \Omega$ ) filled with $2 \mathrm{M} \mathrm{NaCl}$ was placed in stratum radiatum of $\mathrm{CAl}$, but more distal to subfield CA3 than the stimulating electrodes, to detect the population EPSP and afferent volley (Fig. 1A). The amplitude of the population EPSP ranged from 0.5 to 1.5 $\mathrm{mV}$ while that for the afferent volley ranged from 100 to $700 \mu \mathrm{V}$. With the noise level kept below $50 \mu \mathrm{V}$, the signal-to-noise ratios for both the population EPSP and afferent volley were adequate to allow a systematic study of periodic fluctuations in amplitude. Because of the relative size and time course of the population EPSP with respect to that of the afferent volley, however, the simultaneous recording of the afferent volley superimposed upon the population EPSP may introduce an apparent oscillation in the afferent volley arising from oscillations in the population EPSP. To eliminate this distortion, the afferent volley and population EPSP were recorded in separate preparations. The afferent volley was isolated from the population EPSP in 15 slice preparations by temporarily placing the hippocampal slices into a non-aerated glucose Ringer solution. This anoxic condition was found to arrest the process of synaptic transmission, but not the evoked radiatum fiber activity. These slices were then placed in the inner well of the aerated incubation and recording chamber, In 11 other preparations, synaptic transmission was maintained in order to evoke and record population EPSP responses. The detected evoked responses were amplified via a high impedance preamplifier (Transidyne General 1600) and recorded on the FM channels of a HP3960 tape recorder for later processing. Paired stimuli of identical intensity separated by $40 \mathrm{~ms}$ in time were applied once every $2 \mathrm{~s}$. Each recorded evoked response was digitized, stored on a computer disc file (HP21MX). The digitized responses were analyzed to determine the amplitudes of the evoked responses using algorithms to determine the peak negativity of the afferent volley response and the negativity of the population EPSP at a fixed latency ( $1 \mathrm{~ms}$ after the stimulus artifact).

Response amplitudes analyzed as a function of time are illustrated in Fig. 1B. It is evident from this plot that there are time variations in the size of the population EPSPs for both the control (squares) and potentiated (crosses) responses. The time variation of the evoked responses was examined in 11 slices by deternining the time between local maximum and/or minimum values and by frequency analysis using fast 


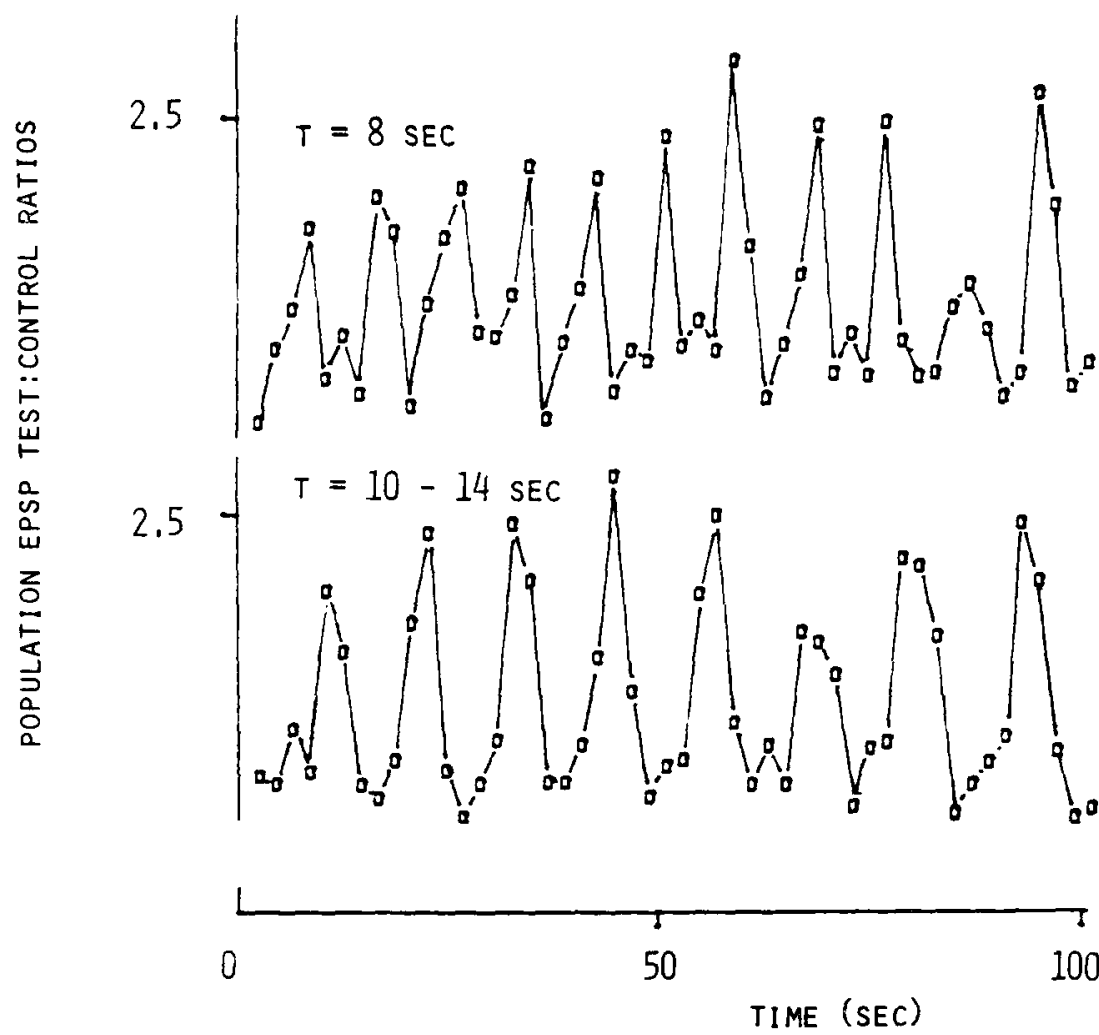

Fig. 2. Modification of oscillations in population EPSP test:control ratio by the electrical activation of radiation fibers. A: oscillations with suprathreshold stimulation. B: alterations in time period of oscillation with suprathreshold stimulation after a brief increase in stimulus intensity.

Fourier transfor ms ${ }^{7}$. Ten of the 11 slices exhibited well developed oscillations of the population EPSP. The period of oscillation for the 10 slices ranged from 8 to $20 \mathrm{~s}$. The period of oscillation for individual slices, however, varied less than $\pm 20 \%$.

It was also noted that control responses of relatively small amplitude were associated with test responses of relatively large amplitude, while control responses of relatively large amplitude were associated with test responses of relatively small amplitude. This relationship is demonstrated by the plot of test :control ratios shown in Fig. 1C, where the maxima are associated with large test response amplitudes and small control response amplitudes and the minima are associated with small test response amplitudes and large control response amplitudes. The time between the maxima for the responses of Fig. 1C ranged from 14 to $16 \mathrm{~s}$ (each response represents an elapsed time of $2 \mathrm{~s}$ ).

The oscillations exhibited by the populations EPSPs raised the question of oscillations in afferent fiber activity as a possible source of this periodic behavior. Fifteen slices were examined for oscillations in isolated radiatum fiber activity. Oscillations in afferent activity and in the test:control ratio of afferent activity with time periods on the order of 8 to $20 \mathrm{~s}$ were not observed among the 15 slices, as 
exemplified by the responses of Fig. 1D and E, respectively. These observations, therefore, suggested that the oscillations of the population EPSP and the test:control ratios are synaptic in nature.

Stimulation of the radiatum fibers was found to affect the amplitude and frequency of the oscillations in the test control ratio. One such example is shown in Fig. 2. Oscillations in the test:control ratio with a time period of $8 \mathrm{~s}$ (Fig. 2, segment A) were obtained with a suprathreshold stimulus of $48 \mu \mathrm{A}$ applied at a frequency of $0.5 \mathrm{~Hz}$. The period of oscillation under these conditions was altered to $10-14 \mathrm{~s}$ (segment B) after briefly stimulating the radiatum fibers with increases in current intensity up to $60 \mu \mathrm{A}$ applied at $0.5 \mathrm{~Hz}$. Similar alterations in time period and amplitude of the test:control ratio were induced by stimulating the radiatum fibers at various frequencies ( 2 through $20 \mathrm{~Hz}$ ). Alterations in oscillation frequency and test:control ratios emphasize the influence of previous synaptic events on synaptic activity. It should be noted, however, that although alterations in either the time period and/or peak test:control ratios of the oscillations were easily induced, specific alterations in response to specific stimulus patterns have yet to be identified.

The studies described in this report document two types of oscillations. We have demonstrated an oscillation of synaptic transmission as indicated by the synchronous increases and decreases in the population EPSP amplitude shown in the control and test responses of Fig. 1B. The second is an oscillation in the facilitation of synaptic transmission as shown in Fig. IC. One might propose that the apparent oscillations in the population EPSP and its test:control ratio could be explained in terms of the interactions between EPSPs and inhibitory postsynaptic potentials (IPSPs) arising from the activation of inhibitory interneurons by way of the discharge of pyramidal cells. If the pyramidal cell population response to the initial stimulus of a stimulus pair is small. then the subsequent activation of inhibitory interneurons would also be small. The resulting IPSPs in turn would be less effective in inhibiting the second response. On the other hand, if the initial pyramidal population response is large, the following EPSP could be shunted by the preceding IPSP. The spatial separation of the excitatory radiatum terminations and the inhibitory synapses upon hippocampal pyramidal cells, however, would make such a mechanism unlikely and difficult to detect with extracellular field recording techniques. Furthermore, the recurrent inhibitory innervation of the pyramidal cells is substantially reduced in transverse hippocampal slice preparations because of the severing of longitudinal fibers projecting from the basket cells.

It seems more likely that the cyclic oscillations may involve periodic changes in transmitter release. Evidence for oscillations in transmitter release has been demonstrated in the frog neuromuscular junction ${ }^{9}$ and in the electric organ of Torped $o^{4,5}$ with time periods of 14 and $5 \mathrm{~s}$, respectively. These time periods correspond well to the oscillations in summated EPSPs found in the hippocampal slice. The periodic fluctuations in ACh levels observed in the electric organ of Torpedo have been shown to correlate with changes in ATP levels which may result from the regulation of enzymes involved in the synthesis of the neurotransmitter, as suggested by Israel et al. ${ }^{5}$.

Oscillations in transmitter release may also be explained in terms of cyclic 
variations of intraterminal $\mathrm{Ca}^{2+}$ from exogenous ${ }^{10}$ and bound endogenous ${ }^{10,11}$ sources, as proposed by Meiri and Rahamimoff 9 . Similarly oscillations in the enhancement of synaptic transmission with its inverse phase relationship between control and test responses may also reflect cyclic variations in $\mathrm{Ca}^{2+}$ and in the number of vesicles available for synaptic transmission. For instance, the application of a control stimulus during high concentrations of intraterminal $\mathrm{Ca}^{2+}$ could result in the release of a significant portion of the available pool of vesicles. Therefore, the application of a test stimulus $40 \mathrm{~ms}$ later would result in the release of the remaining smaller fraction of the available pool of vesicles. On the other hand, low intraterminal concentrations of $\mathrm{Ca}^{2+}$ would result in the release of a moderate portion of the available vesicle pool, by the control stimulus, and therefore, leave a substantial portion of vesicles to be released by the subsequent test stimulus. Regardless of the mechanism(s) involved, however, the cyclic variations in synaptic transmission and the enhancement of synaptic transmission indicate a synchrony of activity which reflect cooperative synaptic actions.

This work was supported by PHS Grants GM 01284 and NS 08470.

1 Andersen, P., Organization of hippocampal neurons and their interconnections, In R. L. Isaacson and K. H., Pribram (Eds.), The Hippocampus, Vol. I, Plenum Press, New York, 1975, pp. 155-176.

2 Andersen, P., Sundberg, S. H., Sveen, O. and Wigstrom, H. H., Specific long-lasting potentiation of synaptic transmission in hippocampal slices, Nature, (Lond.), 266 (1977) 736-737.

3 Del Castillo, J. and Katz, B., Statistical factors involved in neuromuscular facilitation and depression, J. Physiol. (Lond.), 124 (1954) 574-585.

4 Dunant, Y., Jirounek, P., Israel, M., Lesbats, L. and Manaranche, R., Sustained oscillations of acetylcholine during nerve stimulation, Nature (Lond.), 252 (1974) 485-586.

5 Israel, M., Dunant, Y., Lesbats, B., Manaranche, R., Marsal, J., and F. Meunier, Rapid acetylcholine and adenosine triphosphate oscillations triggered by stimulation of the Torpedo electric organ, J. exp. Biol., 63 (1979) 63-73.

$6 \mathrm{Katz}, \mathrm{B}$. and Miledi, R., The statistical nature of the acetylcholine potential and its molecular components, J. Physiol. (Lond.), 224 (1972) 665-699.

7 Low, W. C., Analysis of Neurophysiological Plasticity within the Hippocampal Formation, Ph. D. thesis, University of Michigan Microfilms, Ann Arbor, MI, 1979.

8 Low, W. C. and BeMent, S. L., Enhancement of afferent fiber activity in hippocampal slices, Brain Research, 198 (1980) 472-477.

9 Meiri, H. and Rahamimoff, R., Clumping and oscillations in evoked transmitter release at the frog neuromuscular junction, J. Physiol. (Lond.), 278 (1978) 513-523.

10 Rahamimoff, R., Erulkar, S. D., Alnaes, E., Meiri, H., Rotshenker, S. and Rahamimoff, H., Modulation of transmitter release by calcium ions and nerve impulses, Cold Spr. Harb. Symp. quant. Biol., 40 (1976) 107-116.

11 Rapp, P. E. and Berridge, M. J., Oscillations in calcium-cyclic AMP control loops form the basis of pacemaker activity and other high frequency biological rhythms, I. theor. Biol., 66 (1977) 497-525.

12 Schwartzkroin, P. A. and Webster, K., Long-lasting facilitation of a synaptic potential following tetanization in the in vitro hippocampal slice, Brain Research, 89 (1975) 107-119.

13 Zucker, R. S., Changes in the statistics of transmitter release during facilitation, J. Physiol. (Lond.), 229 (1973) 787-810. 


\title{
Periodic fluctuations in synaptic transmission and enhancement of transmission in hippocampal slices
}

\author{
WALTER C. LOW*, SPENCER L. BEMENT and DAVID WHITEHORN \\ Bioelectrical Sciences Laboratory, University of Michigan, Ann Arbor, MI 48109 and Department of \\ Physiology and Biophysics, University of Vermont, Burlington, VT 05405 (U.S.A.)
}

(Accepted October 8th, 1981)

Key words: periodic fluctuations - synaptic transmission - hippocampal slices

The properties of the synchronously activated radiatum fiber-CAl synaptic population were examined with the in vitro hippocampal slice preparation. Periodic fluctuations in synaptic transmission and in the enhancement of synaptic transmission were observed with periods ranging from 8 to $20 \mathrm{~s}$. Such periodic fluctuations did not arise from fluctuations in afferent radiatum fiber activity. The period and amplitude of the cyclic variations in the enhancement of synaptic transmission were found to be altered with repeated electrical stimulation of the radiatum fibers. These results reflect cooperative synaptic actions which must be taken into consideration in the delineation of the mechanisms of potentiation.

The statistical nature of neurotransmission is now well documented ${ }^{3,6,13}$. Recent studies, however, using frog neuromuscular junction and electric organ of Torpedo marmorata $^{4,5}$ suggest that slow oscillations in the availability and release of neurotransmitters may also occur. We now present evidence for periodic fluctuations in synaptic transmission and in the enhancement of synaptic transmission for a homogeneous population of synapses within the mammalian central nervous system. In hippocampal slices from rat we recorded evoked field potentials that reflect afferent fiber responses and summated dendritic depolarizations (population EPSPs) evoked by the electrical stimulation of afferent radiatum fibers that terminate on the apical dendrites of CAI pyramidal cells $\mathrm{s}^{2,8,12}$. With stimuli delivered at a rate of $0.5 \mathrm{~Hz}$ we found a well developed periodicity in the size of the population EPSP, but not in the afferent radiatum fiber response. With paired stimuli (40 ms interstimulus interval) the response to the second stimuli was generally greater than the first. This facilitation was quantified as a test:control ratio. We found a strong periodicity in the magnitude of the test:control ratios with the period of the oscillations ranging from 8 to $20 \mathrm{~s}$ in different preparations. In any particular slice the periodicity for test:control ratios was similar to that observed for responses to single stimuli. Thus a regular oscillation appears to exist both in synaptic transmission and in the facilitation of transmission which occurs as a result of prior activation.

Hippocampal slices were prepared from rats (Sprague-Dawley, 100-125 g) with methods similar to those previously described ${ }^{7,8}$. Slices were sectioned transverse to

\footnotetext{
* Present address: Department of Physiology and Biophysics, University of Vermont, Burlington, YT 05405, U.S.A.
} 


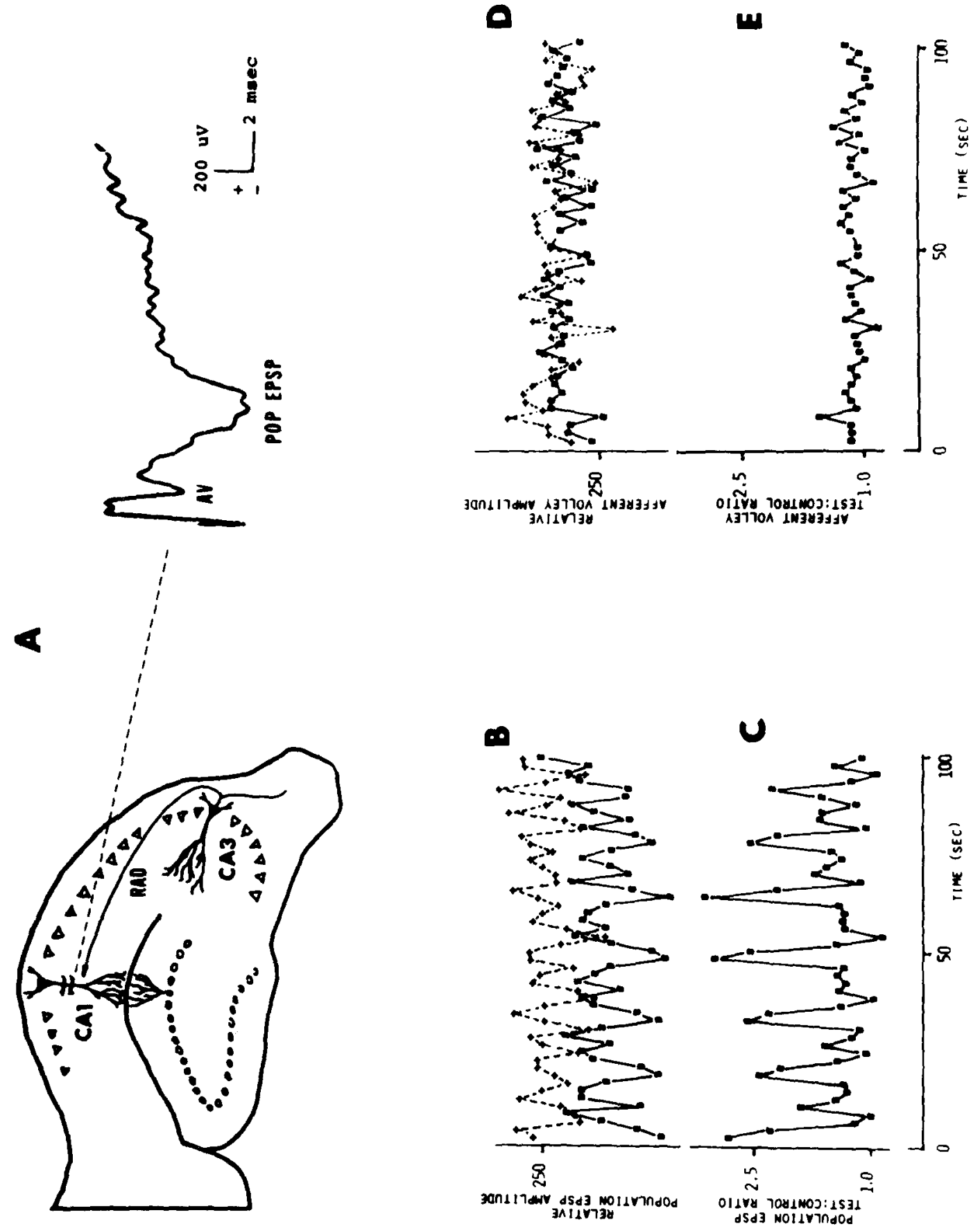

Fig. 1. Oscillation of cvoked population EPSP responses. A: afferent volley (AV) and population EPSP as recorded in stratum radiatum in response to a single stimulus applied to radiatum fibers. $B$ : control (squares) and test (crosses) population EPSP amplitudes in response to paired stimuli ( $40 \mathrm{~ms}$ inter-stimulus interval) applied once every $2 \mathrm{~s}$ with observed oscillations of both test and control population EPSPs. The period of oscillation for the test responses is $8 \mathrm{~s}$. The oscillation for the control responses appear to exhibit two frequency components, one with a period of $8 \mathrm{~s}$ and the other with a period of $16 \mathrm{~s}$. Note the phase relationship $\left(\sim 180^{\circ}\right)$ between the control and test responses. $\mathrm{C}$ : oscillation of the test:control ratio with a major period of approximately $16 \mathrm{~s}$. The large excursion in this ratio reflects the phase relationship noted in Fig. 1B. D: oscillation of test (crosses) and control (squares) afferent volleys. Periodicities are not as distinct as those of the population EPSP and fluctuations appear more random in nature. E: fluctuation of afferent volley test:control ratio. Note that these fluctuations do not exhibit oscillations like that found for the population EPSP in Fig. $1 \mathrm{C}$. 
the septo-temporal axis of the hippocampal formation and immediately immersed in a cooled $\left(\sim 10^{\circ} \mathrm{C}\right)$ and aerated glucose Ringer solution $(124 \mathrm{mM} \mathrm{NaCl}, 5 \mathrm{~m} \mathrm{M} \mathrm{KCl}, 1.24$

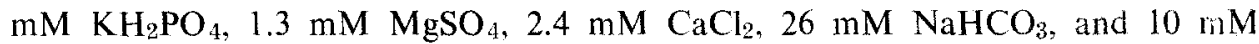
glucose). After sectioning, slices were placed upon a nylon net in the inner well of a warmed $\left(37+0.5^{\circ} \mathrm{C}\right)$ incubation chamber that also contained the glucose Ringer. $A$ gaseous mixture of $95 \% \mathrm{O}_{2}$ and $5 \%, \mathrm{CO}_{2}$ was bubbled into the outer well of the chamber filled with distilled water for approximately $2 \mathrm{~h}$ to create an oxygen gradient which results in the diffusion of $\mathrm{O}_{2}$ into the slices.

Bipolar stimulating electrodes (40-gauge Nichrome) were placed in stratum radiatum of subfield $\mathrm{CAl}$, and an isolated constant current source driven by a Grass stimulator (Model SD5) was used to excite the radiatum fibers with paired current pulses separated in time by $40 \mathrm{~ms}$. Current pulses of $100 \mu$ s duration were used to evoke responses from strata radiatum. The range of current intensities used for the majority of the slices typically varied from 30 to $100 \mu \mathrm{A}$. A recording electrode (1- 3 $\mathrm{M} \Omega$ ) filled with $2 \mathrm{M} \mathrm{NaCl}$ was placed in stratum radiatum of $\mathrm{CAl}$, but more distal to subfield CA3 than the stimulating electrodes, to detect the population EPSP and afferent volley (Fig. 1A). The amplitude of the population EPSP ranged from 0.5 to 1.5 $\mathrm{mV}$ while that for the afferent volley ranged from 100 to $700 \mu \mathrm{V}$. With the noise level kept below $50 \mu \mathrm{V}$, the signal-to-noise ratios for both the population EPSP and afferent volley were adequate to allow a systematic study of periodic fluctuations in amplitude. Because of the relative size and time course of the population EPSP with respect to that of the afferent volley, however, the simultaneous recording of the afferent volley superimposed upon the population EPSP may introduce an apparent oscillation in the afferent volley arising from oscillations in the population EPSP. To eliminate this distortion, the afferent volley and population EPSP were recorded in separate preparations. The afferent volley was isolated from the population EPSP in 15 slice preparations by temporarily placing the hippocampal slices into a non-aerated glucose Ringer solution. This anoxic condition was found to arrest the process of synaptic transmission, but not the evoked radiatum fiber activity. These slices were then placed in the inner well of the aerated incubation and recording chamber, In 11 other preparations, synaptic transmission was maintained in order to evoke and record population EPSP responses. The detected evoked responses were amplified via a high impedance preamplifier (Transidyne General 1600) and recorded on the FM channels of a HP3960 tape recorder for later processing. Paired stimuli of identical intensity separated by $40 \mathrm{~ms}$ in time were applied once every $2 \mathrm{~s}$. Each recorded evoked response was digitized, stored on a computer disc file (HP21MX). The digitized responses were analyzed to determine the amplitudes of the evoked responses using algorithms to determine the peak negativity of the afferent volley response and the negativity of the population EPSP at a fixed latency ( $1 \mathrm{~ms}$ after the stimulus artifact).

Response amplitudes analyzed as a function of time are illustrated in Fig. 1B. It is evident from this plot that there are time variations in the size of the population EPSPs for both the control (squares) and potentiated (crosses) responses. The time variation of the evoked responses was examined in 11 slices by deternining the time between local maximum and/or minimum values and by frequency analysis using fast 


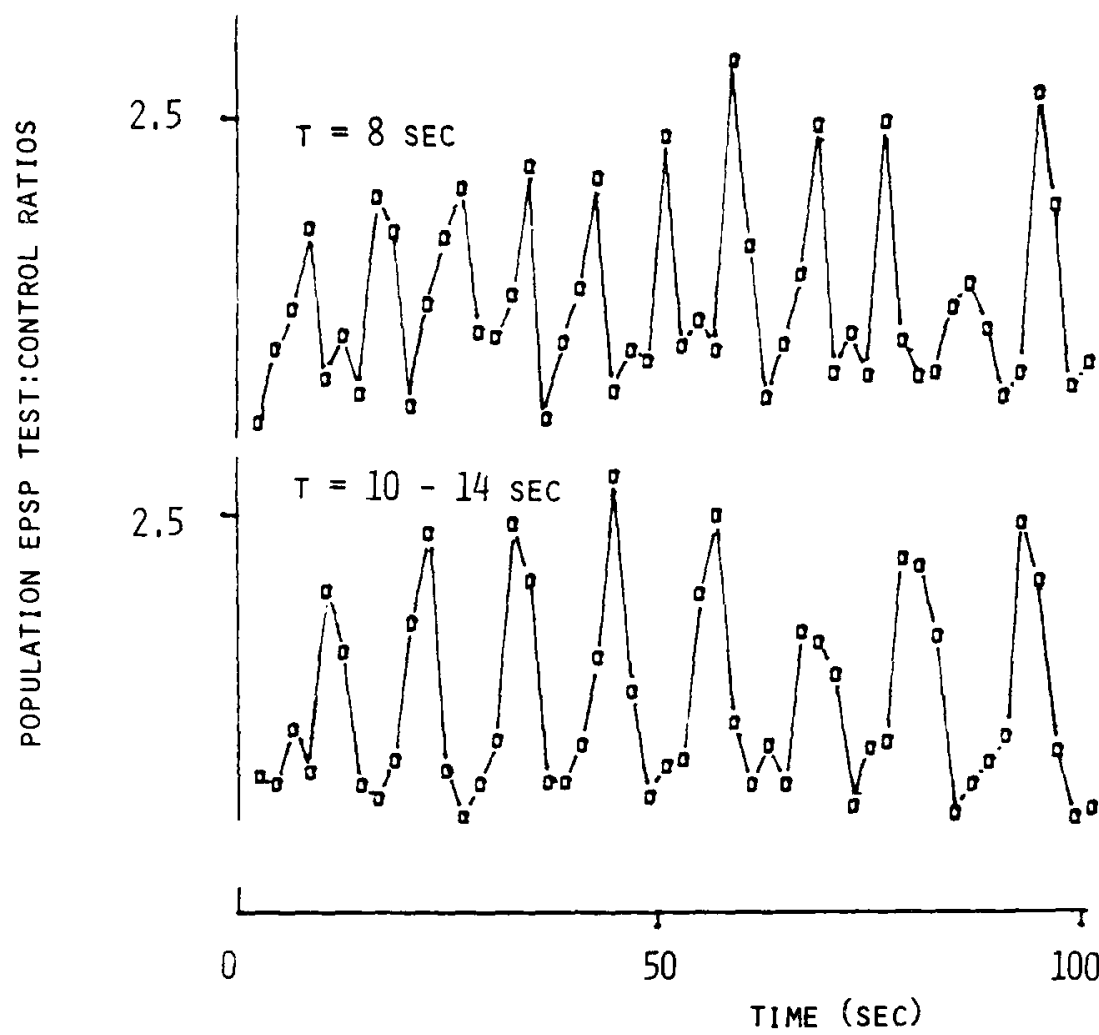

Fig. 2. Modification of oscillations in population EPSP test:control ratio by the electrical activation of radiation fibers. A: oscillations with suprathreshold stimulation. B: alterations in time period of oscillation with suprathreshold stimulation after a brief increase in stimulus intensity.

Fourier transfor ms ${ }^{7}$. Ten of the 11 slices exhibited well developed oscillations of the population EPSP. The period of oscillation for the 10 slices ranged from 8 to $20 \mathrm{~s}$. The period of oscillation for individual slices, however, varied less than $\pm 20 \%$.

It was also noted that control responses of relatively small amplitude were associated with test responses of relatively large amplitude, while control responses of relatively large amplitude were associated with test responses of relatively small amplitude. This relationship is demonstrated by the plot of test :control ratios shown in Fig. 1C, where the maxima are associated with large test response amplitudes and small control response amplitudes and the minima are associated with small test response amplitudes and large control response amplitudes. The time between the maxima for the responses of Fig. 1C ranged from 14 to $16 \mathrm{~s}$ (each response represents an elapsed time of $2 \mathrm{~s}$ ).

The oscillations exhibited by the populations EPSPs raised the question of oscillations in afferent fiber activity as a possible source of this periodic behavior. Fifteen slices were examined for oscillations in isolated radiatum fiber activity. Oscillations in afferent activity and in the test:control ratio of afferent activity with time periods on the order of 8 to $20 \mathrm{~s}$ were not observed among the 15 slices, as 
exemplified by the responses of Fig. 1D and E, respectively. These observations, therefore, suggested that the oscillations of the population EPSP and the test:control ratios are synaptic in nature.

Stimulation of the radiatum fibers was found to affect the amplitude and frequency of the oscillations in the test control ratio. One such example is shown in Fig. 2. Oscillations in the test:control ratio with a time period of $8 \mathrm{~s}$ (Fig. 2, segment A) were obtained with a suprathreshold stimulus of $48 \mu \mathrm{A}$ applied at a frequency of $0.5 \mathrm{~Hz}$. The period of oscillation under these conditions was altered to $10-14 \mathrm{~s}$ (segment B) after briefly stimulating the radiatum fibers with increases in current intensity up to $60 \mu \mathrm{A}$ applied at $0.5 \mathrm{~Hz}$. Similar alterations in time period and amplitude of the test:control ratio were induced by stimulating the radiatum fibers at various frequencies ( 2 through $20 \mathrm{~Hz}$ ). Alterations in oscillation frequency and test:control ratios emphasize the influence of previous synaptic events on synaptic activity. It should be noted, however, that although alterations in either the time period and/or peak test:control ratios of the oscillations were easily induced, specific alterations in response to specific stimulus patterns have yet to be identified.

The studies described in this report document two types of oscillations. We have demonstrated an oscillation of synaptic transmission as indicated by the synchronous increases and decreases in the population EPSP amplitude shown in the control and test responses of Fig. 1B. The second is an oscillation in the facilitation of synaptic transmission as shown in Fig. IC. One might propose that the apparent oscillations in the population EPSP and its test:control ratio could be explained in terms of the interactions between EPSPs and inhibitory postsynaptic potentials (IPSPs) arising from the activation of inhibitory interneurons by way of the discharge of pyramidal cells. If the pyramidal cell population response to the initial stimulus of a stimulus pair is small. then the subsequent activation of inhibitory interneurons would also be small. The resulting IPSPs in turn would be less effective in inhibiting the second response. On the other hand, if the initial pyramidal population response is large, the following EPSP could be shunted by the preceding IPSP. The spatial separation of the excitatory radiatum terminations and the inhibitory synapses upon hippocampal pyramidal cells, however, would make such a mechanism unlikely and difficult to detect with extracellular field recording techniques. Furthermore, the recurrent inhibitory innervation of the pyramidal cells is substantially reduced in transverse hippocampal slice preparations because of the severing of longitudinal fibers projecting from the basket cells.

It seems more likely that the cyclic oscillations may involve periodic changes in transmitter release. Evidence for oscillations in transmitter release has been demonstrated in the frog neuromuscular junction ${ }^{9}$ and in the electric organ of Torped $o^{4,5}$ with time periods of 14 and $5 \mathrm{~s}$, respectively. These time periods correspond well to the oscillations in summated EPSPs found in the hippocampal slice. The periodic fluctuations in ACh levels observed in the electric organ of Torpedo have been shown to correlate with changes in ATP levels which may result from the regulation of enzymes involved in the synthesis of the neurotransmitter, as suggested by Israel et al. ${ }^{5}$.

Oscillations in transmitter release may also be explained in terms of cyclic 
variations of intraterminal $\mathrm{Ca}^{2+}$ from exogenous ${ }^{10}$ and bound endogenous ${ }^{10,11}$ sources, as proposed by Meiri and Rahamimoff 9 . Similarly oscillations in the enhancement of synaptic transmission with its inverse phase relationship between control and test responses may also reflect cyclic variations in $\mathrm{Ca}^{2+}$ and in the number of vesicles available for synaptic transmission. For instance, the application of a control stimulus during high concentrations of intraterminal $\mathrm{Ca}^{2+}$ could result in the release of a significant portion of the available pool of vesicles. Therefore, the application of a test stimulus $40 \mathrm{~ms}$ later would result in the release of the remaining smaller fraction of the available pool of vesicles. On the other hand, low intraterminal concentrations of $\mathrm{Ca}^{2+}$ would result in the release of a moderate portion of the available vesicle pool, by the control stimulus, and therefore, leave a substantial portion of vesicles to be released by the subsequent test stimulus. Regardless of the mechanism(s) involved, however, the cyclic variations in synaptic transmission and the enhancement of synaptic transmission indicate a synchrony of activity which reflect cooperative synaptic actions.

This work was supported by PHS Grants GM 01284 and NS 08470.

1 Andersen, P., Organization of hippocampal neurons and their interconnections, In R. L. Isaacson and K. H., Pribram (Eds.), The Hippocampus, Vol. I, Plenum Press, New York, 1975, pp. 155-176.

2 Andersen, P., Sundberg, S. H., Sveen, O. and Wigstrom, H. H., Specific long-lasting potentiation of synaptic transmission in hippocampal slices, Nature, (Lond.), 266 (1977) 736-737.

3 Del Castillo, J. and Katz, B., Statistical factors involved in neuromuscular facilitation and depression, J. Physiol. (Lond.), 124 (1954) 574-585.

4 Dunant, Y., Jirounek, P., Israel, M., Lesbats, L. and Manaranche, R., Sustained oscillations of acetylcholine during nerve stimulation, Nature (Lond.), 252 (1974) 485-586.

5 Israel, M., Dunant, Y., Lesbats, B., Manaranche, R., Marsal, J., and F. Meunier, Rapid acetylcholine and adenosine triphosphate oscillations triggered by stimulation of the Torpedo electric organ, J. exp. Biol., 63 (1979) 63-73.

$6 \mathrm{Katz}, \mathrm{B}$. and Miledi, R., The statistical nature of the acetylcholine potential and its molecular components, J. Physiol. (Lond.), 224 (1972) 665-699.

7 Low, W. C., Analysis of Neurophysiological Plasticity within the Hippocampal Formation, Ph. D. thesis, University of Michigan Microfilms, Ann Arbor, MI, 1979.

8 Low, W. C. and BeMent, S. L., Enhancement of afferent fiber activity in hippocampal slices, Brain Research, 198 (1980) 472-477.

9 Meiri, H. and Rahamimoff, R., Clumping and oscillations in evoked transmitter release at the frog neuromuscular junction, J. Physiol. (Lond.), 278 (1978) 513-523.

10 Rahamimoff, R., Erulkar, S. D., Alnaes, E., Meiri, H., Rotshenker, S. and Rahamimoff, H., Modulation of transmitter release by calcium ions and nerve impulses, Cold Spr. Harb. Symp. quant. Biol., 40 (1976) 107-116.

11 Rapp, P. E. and Berridge, M. J., Oscillations in calcium-cyclic AMP control loops form the basis of pacemaker activity and other high frequency biological rhythms, I. theor. Biol., 66 (1977) 497-525.

12 Schwartzkroin, P. A. and Webster, K., Long-lasting facilitation of a synaptic potential following tetanization in the in vitro hippocampal slice, Brain Research, 89 (1975) 107-119.

13 Zucker, R. S., Changes in the statistics of transmitter release during facilitation, J. Physiol. (Lond.), 229 (1973) 787-810. 